\title{
Three-dimensional Planning in Orthognathic Surgery using Cone-beam Computed Tomography and Computer Software
}

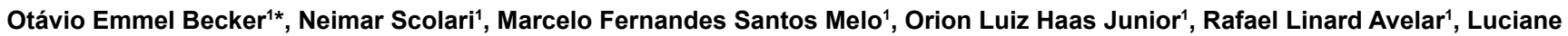
Macedo De Menezes ${ }^{2}$ and Rogério Belle De Oliveira ${ }^{1}$

${ }^{1}$ Oral and Maxillofacial Surgery Department, Pontifical Catholic University of Rio Grande do Sul, Porto Alegre, Brazil

${ }^{2}$ Orthodontics Department, Pontifical Catholic University of Rio Grande do Sul, Porto Alegre, Brazil

\begin{abstract}
The orthognathic surgery is the standard treatment for the correction of dentofacial deformities, in order to get a stable dental occlusion and facial harmony. The advancement of technology and the evolution of the concepts involved in the diagnosis and treatment plan in this area have been immeasurable, leading to the development of new methods, such as computer-aided jaw surgery system by a three-dimensional (3D) virtual surgical planning. The advent of the cone-beam computed tomography (CBCT) allows the acquisition of 3D images of the patient's craniofacial complex and eliminates the ambiguity that can occur with two-dimensional (2D) images. Surgical simulation in 3D may benefit patients by providing a more accurate treatment plan and streamlined surgery, especially for patients with complex dentofacial deformities. The breakthrough of software tools for the diagnosis and treatment planning allows the construction of 3D surface models, dynamic cephalometry, semi-automatic mirroring in cases of asymmetry, interactive cutting of bone, bony segment repositioning, 3D splint manufacturing, bone reconstruction and visualization and prediction of the changes in hard and soft tissues of the face. The aim of this study was to report a case where the computed-assisted surgical planning predicts the possibility in achieving balance between aesthetic and function. Alterations in the virtual planning allow overcoming obstacles in actual surgery. Considering all possible details, the process provides greater predictability, practicality and precision in surgical planning.
\end{abstract}

Keywords: Orthognathic surgery; Virtual planning; Computer aided systems; Dentofacial deformities; Cone beam computed tomography

Abbreviations: CBCT: Cone-Beam Computed Tomography (CBCT); 3D: Three-Dimensional (3D); 2D: Two-Dimensional (2D); DICOM: Digital Imaging and Communications in Medicine (DICOM)

\section{Introduction}

The orthognathic surgery is the standard treatment for the correction of dentofacial deformities, in order to get a stable dental occlusion and facial harmony [1-3]. The integration between orthodontics and surgery is one of the pillars of this treatment, and the others are appropriate facial analysis, imaging exams and surgical technique $[4,5]$.

Conventional methods of planning orthognathic surgery depend on two-dimensional (2D) images, including digital photographs and cephalometric and panoramic radiographs to determine discrepancies and craniofacial deformities. These exams provide only a limited understanding of the complex three-dimensional defects, and are insufficient for planning appropriate corrections [1-3,6-9]. This analysis is based on a series of cephalometric landmarks that can be difficult to found due to wrong position of the patient's head during aquisition of the $\mathrm{x}$-rays, the superposition of anatomical structures and differential magnification of bilateral structures, resulting in image distortion and increasing the risk of error [4,5,9-12].

The advent of the cone-beam computed tomography (СBCT) allows the acquisition of three-dimensional (3D) images of the patient's craniofacial complex. The more common use of CBCT requires changes in our methods of diagnosis and treatment planning, as well as additional training $[7,12,13]$. The management of $3 \mathrm{D}$ images differ from conventional $2 \mathrm{D}$ images and eliminates the ambiguity that can occur with them $[6,14]$. The standard for digital computed tomographic images is called Digital Imaging and Communications in Medicine (DICOM), that includes information regarding the patient and the $3 \mathrm{D}$ СBCT, allowing handling, measurement and analysis of the data set using specify softwares [13]. The image can be enhanced and augmented to assist landmark location, thereby minimizing errors. Since changes in soft tissue are directly related to the hard tissue changes, it is important that the system accurately measures hard tissue changes, thus predicting soft tissue changes. Clinical usefulness of the softwares is determined by accurate prediction of the hard and soft tissues $[5,11]$.

The surgical planning and simulation include the construction of 3D surface models, dynamic cephalometry, semi-automatic mirroring in cases of asymmetry, interactive cutting of bone, bony segment repositioning and bone reconstruction [7,9]. Once established, the virtual 3D treatment plan, using computer-aided design and computeraided manufacturing techniques, 3D virtual surgical splints can be processed and used during the actual surgery [15-17].

The promising advances in this field have enabled both simplification of surgical techniques and a more accurate orthodonticsurgical planning, optimizing treatment outcome of maxillofacial deformities $[8,9]$. This work aims to demonstrate that the digital surgical planning allows the surgeon to manipulate and operate digital representations of skull for prediction of orthognathic surgery.

*Corresponding author: Otávio Emmel Becker, Oral and Maxillofacial Surgery Department, Pontifical Catholic University of Rio Grande do Sul, Porto Alegre, Brazil, Tel: 55-48-3224-2623; E-mail: beckerotavio@hotmail.com

Received September 19, 2013; Accepted October 14, 2013; Published October 20, 2013

Citation: Becker OE, Scolari N, Melo MFS, Haas Junior OL, Avelar RL, et al (2013) Three-dimensional Planning in Orthognathic Surgery using Cone-beam Computed Tomography and Computer Software. J Comput Sci Syst Biol 6: 311 316. doi:10.4172/jcsb.1000127

Copyright: (c) 2013 Becker OE, et al. This is an open-access article distributed under the terms of the Creative Commons Attribution License, which permits unrestricted use, distribution, and reproduction in any medium, provided the original author and source are credited. 
Citation: Becker OE, Scolari N, Melo MFS, Haas Junior OL, Avelar RL, et al. (2013) Three-dimensional Planning in Orthognathic Surgery using Conebeam Computed Tomography and Computer Software. J Comput Sci Syst Biol 6: 311-316. doi:10.4172/jcsb.1000127

Therefore, jaw surgery assisted by computer programs provides greater predictability of difficulties and precision in planning, considering all possible details, aiming to balance aesthetic and function in patients. This is shown in the case reported where the computed-assisted surgical planning predicts the possibility in achieving this balance. Alterations in the virtual planning allow overcoming obstacles in actual surgery.

\section{Materials and Methods/Case Report}

Female patient, 29 years, presents dentofacial deformity, Class II facial profile and vertical maxillary excess, Angle's Class I occlusion. The patient's main complaint was the gummy smile and the retroposition of the chin (Figure 1 and 2). To realize the virtual surgical planning, it was performed a CBCT in the i-CAT tomograph (Imaging Sciences International, Hatfield, PA, USA), with 14 bits grayscale, with the aquisition protocol of 40 seconds, with 0,25 voxels, with $13 \mathrm{~cm}$ of FOV (field of view). The images were constructed in 3D in the Dolphin Imaging software (Dolphin Imaging and Management Solutions, Chatsworth, CA, USA) (Figure 3). The software provides a sequence for locating the main cephalometric points of multiple dental, skeletal and soft-tissue land marks. This can be done both in $2 \mathrm{D}$ or $3 \mathrm{D}$ images. The image can be enhanced and enlarged to aid in landmark location. The program demonstrates landmarks expected position, thereby minimizing errors in landmark definition. After the indication of the determined points, the software connects the points to form a recognizable image of the tracing, which may be adjusted manually, if necessary. Selection analysis was then chosen.

This software allows the customization of a new analysis from measurements of existing analyses or the creation of new measurements according to the professional's need. Dolphin Imaging software (Dolphin Imaging and Management Solutions, Chatsworth, CA, USA) presents a set of $3 \mathrm{D}$ tools that allows the orientation of the $\mathrm{CBCT}$, building of $\mathrm{x}$-rays based on CBCT, measurements of hard and soft tissues and superimposition of images. This software has a tool named Orthognathic Surgical Planning that simulates the osteotomies, bone movements and prediction of soft tissues. So, it could be programmed the bilateral mandible sagittal split osteotomy, Le Fort I osteotomy and horizontal chin osteotomy, allowing a predictive vision of where hard and soft tissue would look like (Figure 4 and 5).The virtual surgical planning was based on the patient's complaints, facial analysis and $\mathrm{r}$-rays and CBCT cephalometric analysis, and consisted of bimaxillary advancement of $5 \mathrm{~mm}$, maxillary impaction of $5 \mathrm{~mm}$ and chin advancement of $6 \mathrm{~mm}$ (Figure 6).

After orthognathic surgery, it was requested a CBCT to compare the preoperative and postoperative period. Then an superimposition of the $3 \mathrm{D}$ preoperative and postoperative CBCTs was performed, showing what actually has changed after surgery (Figure 7 and 8). In addition to bone images, it was able to visualize the impact of the surgical procedure in the patient's airway, presenting a considerable gain in the oropharyngeal space (Figure 8 ). The airway volume before surgery was of $15004.4 \mathrm{~mm}^{3}$ and the minimum axial area $109.6 \mathrm{~mm}^{2}$. After surgery, these measurements increased to $28091.00 \mathrm{~mm}^{3}$ and $217.6 \mathrm{~mm}^{2}$ (Figure 9 and 10). In comparison, predictive images of both soft and hard tissues show great similarity with post-operactive images (Figure 11 and 12).

\section{Discussion}

The diagnosis of skeletal discrepancies is based on data from different sources: clinical examination, photographs, CT scans and dental models [18]. Traditional assessment was performed manually using frontal and lateral cephalometric radiographs $[9,12,19]$. However, nowadays computer aided systems integrate different data in

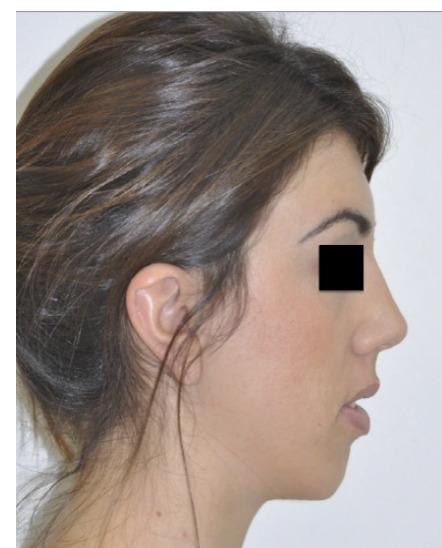

Figure 1: Facial profile of the patient with Class II dentofacial deformity, vertical maxillary excess and retroposition of the chin.

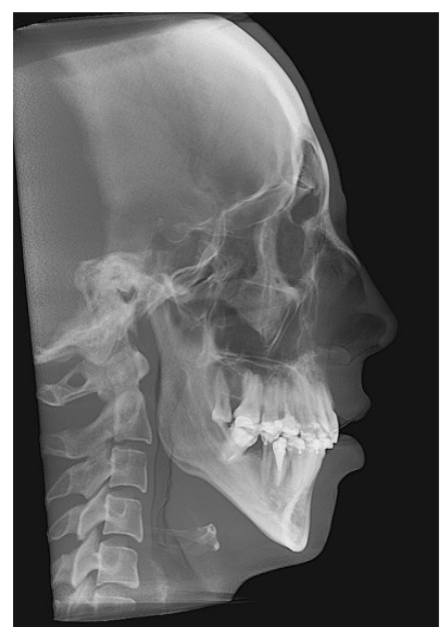

Figure 2: Lateral view of the patient skull showing the Class II dentofacial deformity, retroposition of the chin and Angle's Class I occlusion.

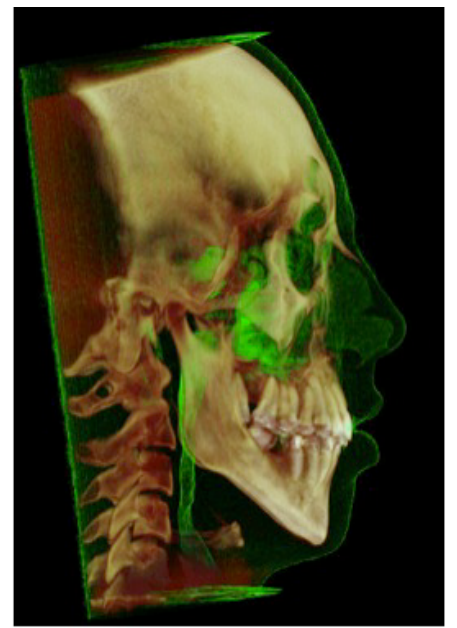

Figure 3: CBCT image reconstructed in 3D in the Dolphin Imaging software showing hard and soft tissues (Dolphin Imaging and Management Solutions, Chatsworth, CA, USA). 
Citation: Becker OE, Scolari N, Melo MFS, Haas Junior OL, Avelar RL, et al. (2013) Three-dimensional Planning in Orthognathic Surgery using Conebeam Computed Tomography and Computer Software. J Comput Sci Syst Biol 6: 311-316. doi:10.4172/jcsb.1000127

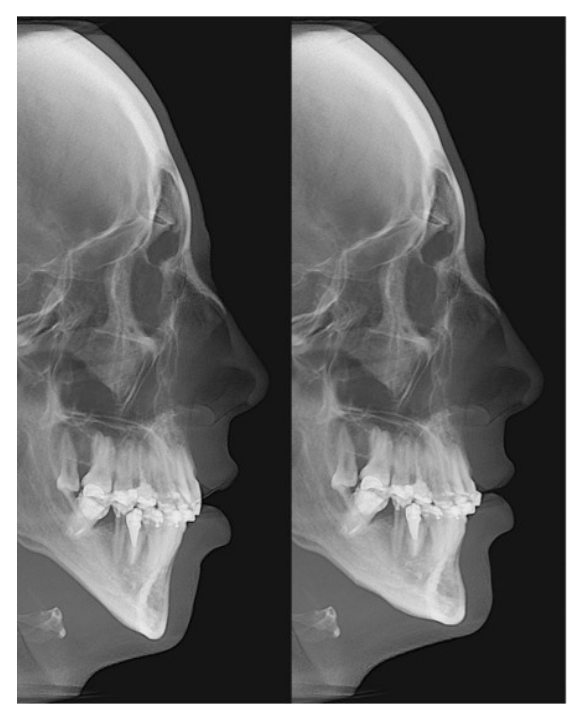

Figure 4: Comparison of hard tissues between pre-operative (left) and predictive (right) views after virtual surgery.

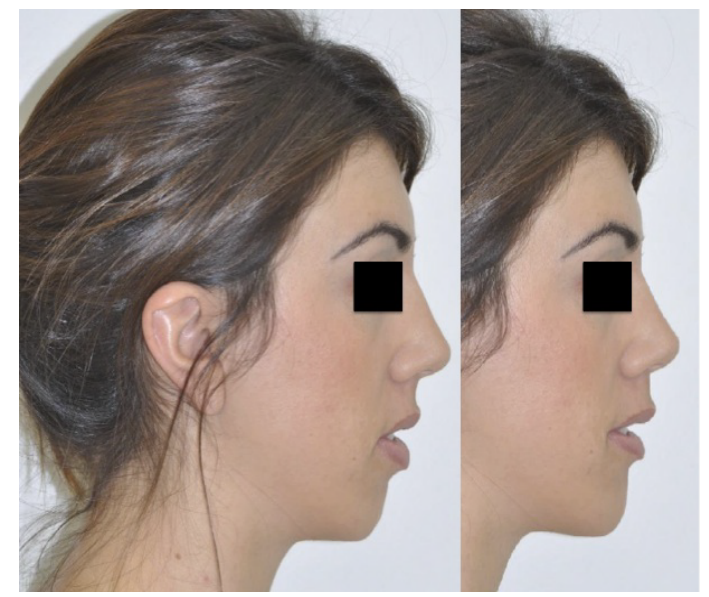

Figure 5: Comparison of soft tissues between pre-operative (left) and predictive (right) views after virtual surgery.

three dimensions and have been developed and used to analyze, plan and predict the outcome of orthognathic surgeries $[9,12,20]$. Threedimensional cephalometric is a valuable tool for diagnosis of skeletal imbalance, to evaluate the growth response to treatment and long-term stability $[12,18,19]$.

There was an explosion in recent years of commercially available programs for 3D virtual surgery and visualization programs. Researches demonstrate the potential of 3D virtual planning for comparable or better surgical outcomes than conventional plannings [8,9,21,22]. The biggest draw-back to these programs is the lack of validation of outcomes. It is desirable that craniofacial skeletal components, occlusion and soft tissue outcomes are validated [21]. In spite of that, three-dimensional surgical planning allows us to overcome many of the limitations of conventional surgical planning, such the lack of prediction of soft-tissues and bone movements three-dimensionally, the necessity of models and articulators and more available time $[21,22]$.
These software programs are developed to allow oral and maxillofacial surgeons to perform orthognathic surgery virtually $[8,23]$. Currently, a number of software programs are available: 3DMD Vultus (3DMD, Atlanta, GA, USA), Maxilim (Medicim, Mechelen, Belgium), Dolphin Imaging (Dolphin Imaging \& Management Solutions, Chatsworth, CA, USA), In vivo Dental (Anatomage, San Jose, CA, USA), SimPlant OMS (Materializa, Leuven, Belgium), ITK-SNAP (open source software, www.itk-snap.org) [18]. Dolphin Imaging (Dolphin Imaging and Management Solutions, Chatsworth, CA, USA) is one of the most used by surgeons and clinicians worldwide.

When using $3 \mathrm{D}$ planning, all the necessary information is

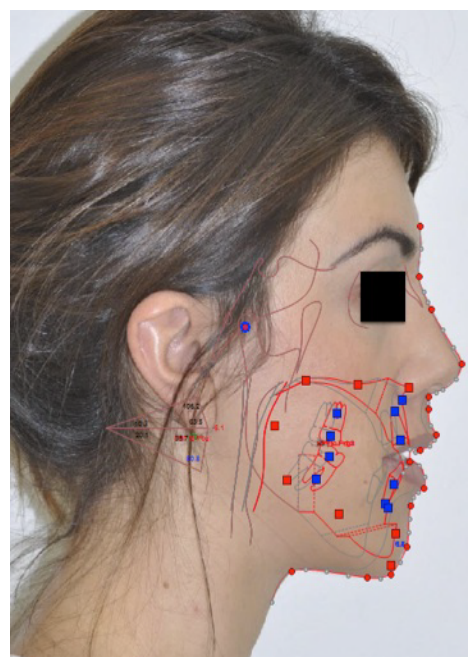

Figure 6: Virtual surgical planning: bimaxillary advancement of $5 \mathrm{~mm}$, maxillary impaction of $5 \mathrm{~mm}$ and chin advancement of $6 \mathrm{~mm}$. Gray tracing shows original position of the jaws and red position after bone movements. Soft tissues follow these movements.

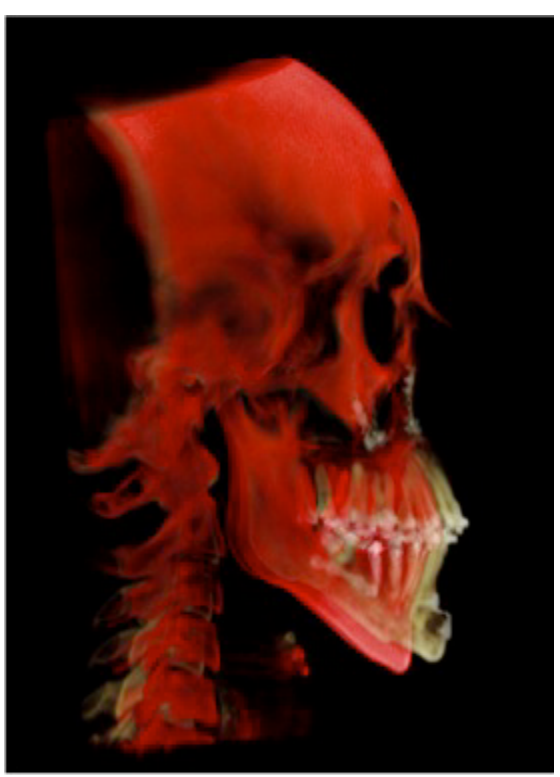

Figure 7: Superimpostion of the 3D preoperative (red)and postoperative (yellow) CBCTs showing what actually has changed after surgery in hard tissues. 
Citation: Becker OE, Scolari N, Melo MFS, Haas Junior OL, Avelar RL, et al. (2013) Three-dimensional Planning in Orthognathic Surgery using Conebeam Computed Tomography and Computer Software. J Comput Sci Syst Biol 6: 311-316. doi:10.4172/jcsb.1000127

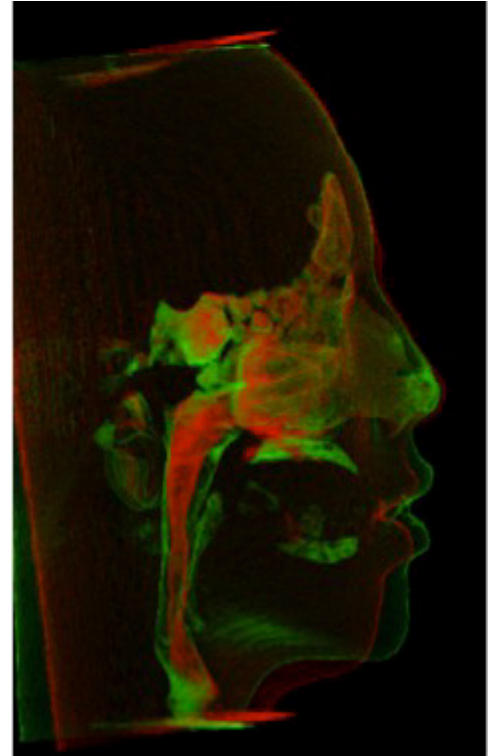

Figure 8: Superimposition of the 3D preoperative (red) and postoperative (green) CBCTs showing what actually has changed after surgery in facial soft tissues and the impact in the patient's airway, presenting a considerable gain in the oropharyngeal space.

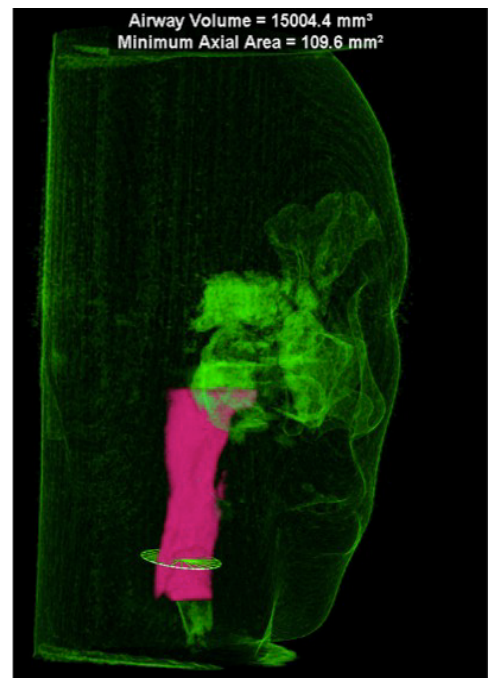

Figure 9: Airway volume and minimum axial area before surgery.

provided in images which can be manipulated on a computer, whilst conventional planning makes it necessary to obtain data from different studies (radiographs, models and articulators, face bow, etc.), and to interpret the data before being able to develop a treatment plan $[9,24]$.

Using this methodology, physical elements used for treatment planning can be eliminated from clinical practice. Treatment plans can be stored online and the space normally taken up by materials used in conventional planning, saved [24]. Data can be stored in one location and analyzed and viewed in different locations, such as at surgical rooms as a transoperative guide. Also, a technician following the instructions of the surgeon can use it. Software tools allow the preparation of detailed surgical plannings as repositioning osteotomies, bone reconstruction, surgery training for residents and assessment of difficulties of surgical procedures before actual surgery $[9,18,22,24,25]$.

These preoperative evaluations and measurements provide the surgeon with $3 \mathrm{D}$ information that will translate into shortening surgical procedures and minimizing potential surgical risks and complications $[23,25]$. The effects of surgical movements upon the soft tissues can be ascertained. Being able to predict postoperative results is a great advantage of a 3D treatment planning system, considering the large number of patients undergoing this type of surgery for aesthetic reasons [24]. By superimposition of photographs, patients can be given an idea of surgical outcome. These data cannot be provided by model surgery in articulators or 2D analysis. Prior to computerized cephalometrics, prediction involved either alteration of the manual tracings or sectioning of lateral photographs, both methods being time-consuming, unrealistic and inaccurate. Working in $3 \mathrm{D}$ enables surgeons to get nearer to the reality with which they have to work in the operating room. The mobilization of osteotomized bone structures is

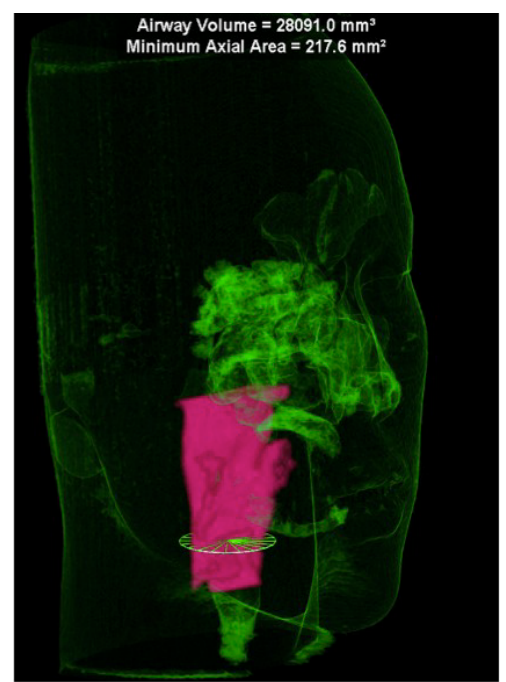

Figure 10: Airway volume and minimum axial area after surgery.

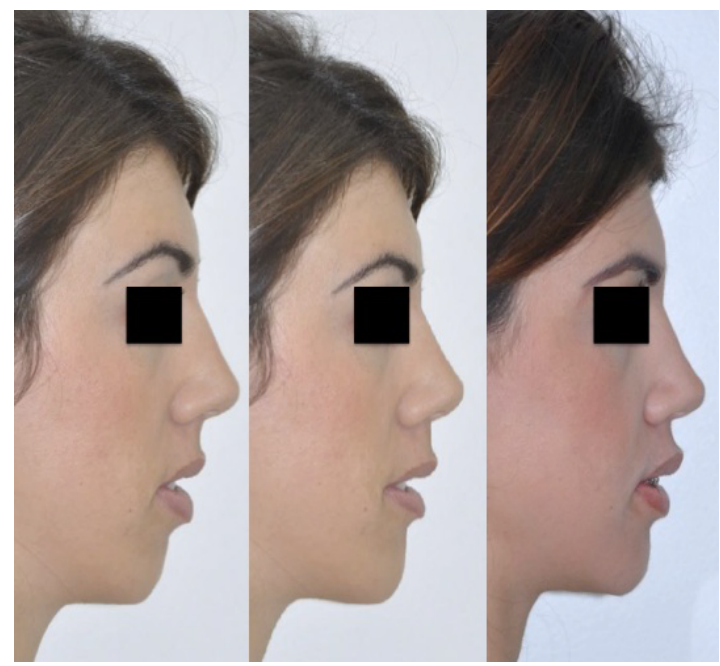

Figure 11: Comparison of soft tissues among pre-operative (left), predictive (center) and post-operative (right) views after surgery. 
Citation: Becker OE, Scolari N, Melo MFS, Haas Junior OL, Avelar RL, et al. (2013) Three-dimensional Planning in Orthognathic Surgery using Conebeam Computed Tomography and Computer Software. J Comput Sci Syst Biol 6: 311-316. doi:10.4172/jcsb.1000127

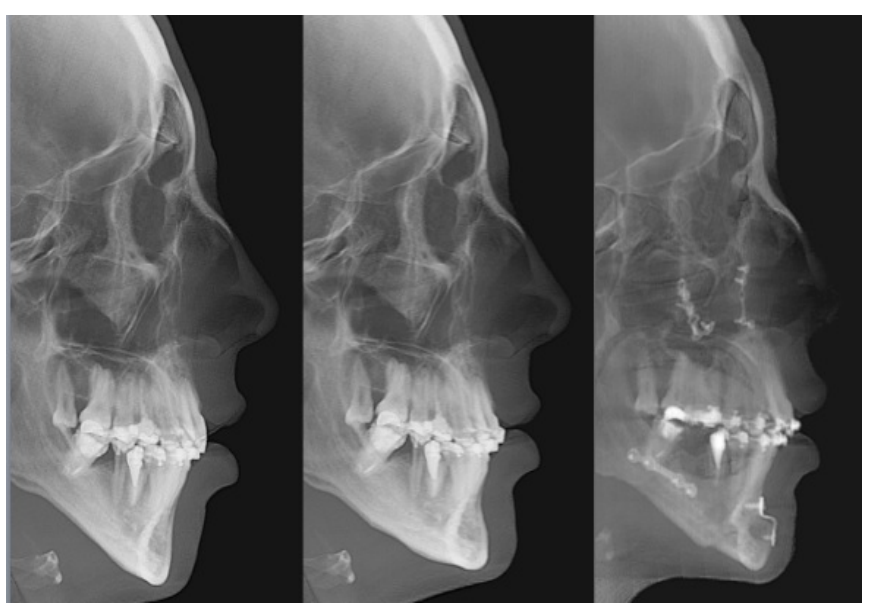

Figure 12: Comparison of hard tissues among pre-operative (left) predictive (center) and post-operative (right) views after surgery.

complex, since it combines rotation and translation in three planes [2224]. The actual procedure and anteroposterior and vertical movements required, can be decided previously. This situation allows the surgeon to have a better idea of how much bone removal will be necessary and then plan accordingly [21].

The softwares can provide a more precise vision of how the condyle will rotate when the mandible is mobilized. An advantage is that the condyle fossa relationship in centric relation remains stable throughout the planning process $[23,24]$. Detailed measurements of the teeth and the anatomic relations of vital structures can be made using the measurement tools found in these software programs [23].

Patients with severe asymmetry can be treated using this new technology. The maxillomandibular complex can be aligned in the correct position and the skeletal asymmetry then corrected. It enables asymmetry of the maxillomandibular complex to be compared with the rest of the facial skeleton in different views so that the anatomical region causing the facial asymmetry can be seen easily $[9,12,17,24]$. The diagnosis can be shared with the patient using a 3D image that can be easily understood [24].

The programs not only assist in making diagnoses, but it also facilitates the manufacture of physical elements, such as stereolithographic surgical splints, which are used during actual surgery. These surgical splints are vital in orthognathic surgery, if predictable results are to be obtained. This is, particularly, so in bimaxillary surgery where complex movements are performed in the three planes $[17,21-$ 26].

Thus, it is demonstrated that computer-aided surgical simulation has lower material costs, the potential to greatly reduce intraoperative time, complications and surgical surprise $[8,21,22]$. Also, it increases predictability. It allows the surgeon to better predict possible surgical complications and adapt accordingly to reduce potential difficulties $[8,21]$. It has also been used to allow more complex surgeries to be successfully performed in a single procedure rather than the previous multiple staged surgeries [21]. In the case described, the computer-aided surgical simulation has predicted the possibility in achieving balance between aesthetic and function. The software allowed the modification of the position of the gnathic bones, improving the surgery outcome by prediction of modification in airway, hard and soft tissues. Predictive images of virtual surgery showed great similarity with post-operactive images after actual surgery.

\section{References}

1. Lu CH, Ko EWC, Huang CS (2003) The accuracy of video imaging prediction in soft tissue outcome after bimaxillary orthognathic surgery. Int J Oral Maxillofac Surg 61: 333-342.

2. Kaipatur NR, Flores-Mir C (2009) Accuracy of computer programs in predicting orthognathic surgery soft tissue response. Int J Oral Maxillofac Surg 67:751759.

3. Sonego CL, Bobrowski ÂN, Junior OLC, Torriani MA (2013) Aesthetic and functional implications following rotation of the maxillomandibular complex in orthognathic surgery: A systematic review. Int J Oral Maxillofac Surg 14: 1-6.

4. Becker OE, Avelar RL, do N Dolzan A, Haas OL Jr, Scolari N, et al. (2013) Soft and hard tissue changes in skeletal Class III patients treated with doublejaw orthognathic surgery-Maxillary advancement and mandibular setback. Int J Oral Maxillofac Surg Jul 16

5. Ahmad Akhoundi MS, Shirani G, Arshad M, Heidar H, Sodagar A (2012) Comparison of an imaging software and manual prediction of soft tissue changes after orthognathic surgery. J Dent (Tehran) 9: 178-187.

6. Caloss R, Atkins K, Stella JP (2007) Three-dimensional imaging for virtua assessment and treatment simulation in orthognathic surgery. Oral Maxillofac Surg Clin North Am 19: 287-309.

7. Cevidanes LHC, Tucker S, Styner M, Kim H, Chapuis J, et al. (2010) Threedimensional surgical simulation. Am J Orthod Dentofacial Orthop 138: 361-371.

8. Xia JJ, Shevchenko L, Gateno J, Teichgraeber JF, Taylor TD, et al. (2011) Outcome study of computer-aided surgical simulation in the treatment of patients with craniomaxillofacial deformities. Int J Oral Maxillofac Surg 69 : 2014-2024.

9. Gateno J, Xia JJ, Teichgraeber JF (2011) New methods to evaluate craniofacia deformity and to plan surgical correction. Semin Orthod 17: 225-234.

10. Ludlow JB, Gubler M, Cevidanes L, Mol A (2009) Precision of cephalometric landmark identification: Cone-beam computed tomography vs conventional cephalometric views. Am J Orthod Dentofacial Orthop 136: 312.e1-312.e10.

11. Power G, Breckon J, Sherriff M, McDonald F (2005) Dolphin imaging software: An analysis of the accuracy of cephalometric digitization and orthognathic prediction. Int J Oral Maxillofac Surg 34: 619-626.

12. Gateno J, Xia JJ, Teichgraeber JF (2011) New 3-dimensional cephalometric analysis for orthognathic surgery. Int J Oral Maxillofac Surg 69: 606-622.

13. Grauer D, Cevidanes LSH, Proffit WR (2009) Working with DICOM craniofacial images. Am J Orthod Dentofacial Orthop 136: 460-470.

14. Cevidanes LHS, Styner MA, Proffit WR (2006) Image analysis and superimposition of 3-dimensional cone-beam computed tomography models. Am J Orthod Dentofacial Orthop 129: 611-618.

15. Gateno J, Xia J, Teichgraeber JF, Rosen A, Hultgren B, et al. (2003) The precision of computer-generated surgical splints. Int J Oral Maxillofac Surg 61 814-817.

16. Swennen GRJ, Mollemans W, Schutyser F (2009) Three-dimensional treatment planning of orthognathic surgery in the era of virtual imaging. Int $\mathrm{J}$ Oral Maxillofac Surg 67: 2080-2092.

17. Xia JJ, Gateno J, Teichgraeber JF (2009) New Clinical protocol to evaluate craniomaxillofacial deformity and plan surgical correction. Int J Oral Maxillofac Surg 67: 2093-2106.

18. Cevidanes LHC, Tucker S, Styner M, Kim H, Chapuis J, et al. (2010) Threedimensional surgical simulation. Am J Orthod Dentofacial Orthop 138: 361-371.

19. Waitzman AA, Posnick JC, Armstrong DC, Pron GE (1992) Craniofacial skeletal measurements based on computed tomography: Part II. Normal values and growth trends. Cleft Palate Craniofac 29: 118-128.

20. Kolokitha OE, Topouzelis N (2011) Cephalometric methods of prediction in orthognathic surgery. J Maxillofac Oral Surg 10: 236-245.

21. Tucker S, Cevidanes LHS, Styner M, Kim H, Reyes M, et al. (2010) Comparison of actual surgical outcomes and 3-dimensional surgical simulations. Int J Oral Maxillofac Surg 68: 2412-2421.

22. Xia JJ, Gateno J, Teichgraeber JF (2005) Three-dimensional computer-aided surgical simulation for maxillofacial surgery. Atlas Oral Maxillofac Surg Clin North Am 13: 25-39. 
Citation: Becker OE, Scolari N, Melo MFS, Haas Junior OL, Avelar RL, et al. (2013) Three-dimensional Planning in Orthognathic Surgery using Conebeam Computed Tomography and Computer Software. J Comput Sci Syst Biol 6: 311-316. doi:10.4172/jcsb.1000127

23. Orentlicher G, Goldsmith D, Horowitz A (2010) Applications of 3-dimensional virtual computerized tomography technology in oral and maxillofacial surgery: Current therapy. Int J Oral Maxillofac Surg 68: 1933-1959.

24. Centenero SAH, Hernández-Alfaro F (2012) 3D planning in orthognathic surgery: CAD/CAM surgical splints and prediction of the soft and hard tissues results-Our experience in 16 cases. J Craniomaxillofac Surg 40: 162-168.
25. Xia JJ, Shevchenko L, Gateno J, Teichgraeber JF, Taylor TD, et al. (2011) Outcome study of computer-aided surgical simulation in the treatment of patients with craniomaxillofacial deformities. Int J Oral Maxillofac Surg 69: 2014-2024.

26. Li B, Zhang L, Sun H, Yuan J, Shen SGF, et al. (2013) A novel method of computer aided orthognathic surgery using individual CAD/CAM templates: $\mathrm{A}$ combination of osteotomy and repositioning guides. $\mathrm{Br} \mathrm{J}$ Oral Maxillofac Surgons Apr 5. 